\title{
On the Convex Hull of Uniform Random Points in a Simple $d$-Polytope*
}

\author{
Fernando Affentranger and John A. Wieacker \\ Mathematisches Institut, Albert-Ludwigs-Universität, Albertstrasse 23b, \\ D.-7800 Freiburg i. Br., Germany \\ Communicated by Rolf Schneider
}

\begin{abstract}
Denote the expected number of facets and vertices and the expected volume of the convex hull $P_{n}$ of $n$ random points, selected independently and uniformly from the interior of a simple $d$-polytope by $E_{n}(f), E_{n}(v)$, and $E_{n}(V)$, respectively. In this note we determine the sharp constants of the asymptotic expansion of $E_{n}(f), E_{n}(v)$, and $E_{n}(V)$, as $n$ tends to infinity. Further, some results concerning the expected shape of $P_{n}$ are given.
\end{abstract}

\section{Introduction}

Let $P$ be a convex polytope in Euclidean space $\mathbb{R}^{d}(d \geqq 1)$ with positive volume $V(P), A$ the $\sigma$-algebra of all Borel subsets of $P$, and $\mu$ the restriction of $V(P)^{-1} \lambda_{d}$ to $\mathscr{A}$, where $\lambda_{d}$ is Lebesque measure in $\mathbb{R}^{d}$. For any integer $n \geqq d+1$, the convex hull of $n$ independent uniform random points in $P$, i.e., the measurable map $P_{n}:\left(X_{1}, \ldots, X_{n}\right) \mapsto \operatorname{conv}\left\{X_{1}, \ldots, X_{n}\right\}$ from the probability space $\left(P^{n}, \mathscr{A}^{n \otimes}, \mu^{n \otimes}\right)$ into the space $\mathscr{P}$ of convex polytopes in $\mathbb{R}^{d}$ (where conv denotes the convex hull and $\mathscr{P}$ is endowed with the Hausdorff metric), is almost surely a simplicial $d$-polytope with at most $n$ vertices. For any measurable functional $\psi$ on the space $\{Q \in \mathscr{P} \mid Q \subset P\}$ let $E_{n}(\psi)$ be the expected value of $\psi \circ P_{n}$. In the following our main interest concerns the cases in which $\psi$ is the number $f$ of facets $((d-1)-$ dimensional faces), the number $v$ of vertices, and the volume $V$. All asymptotic expressions appearing in the text refer to $n \rightarrow \infty$.

\footnotetext{
* The work of F. Affentranger was supported by the Swiss National Foundation.
} 
For $d=2$, the asymptotic behavior of $E_{n}(f)=E_{n}(v)$ and $E_{n}(V)$ was first studied in two classical papers [14], [15] by Rényi and Sulanke. For instance, for a polygon with $r$ vertices they prove

$$
E_{n}(f)=\frac{2 r}{3}(\log n+C)+C_{1}(P)+o(1),
$$

where $C$ is Euler's constant and $C_{1}(P)$ is a constant depending on $P$. A detailed investigation of the planar case was given later by Buchta [4].

In higher dimensions $(d \geqq 3)$, only a few results have been established. Buchta [6] proved that

$$
E_{n}(v) \sim \frac{3}{4} \log ^{2} n
$$

for a tetrahedron in $\mathbb{R}^{3}$. Recalling that $P_{n}$ is simplicial and taking into account Euler's polyhedron theorem, the asymptotic behavior of $E_{n}(f)$ can be directly obtained from $E_{n}(v)$. In the general case estimates for $E_{n}(f), E_{n}(v)$, and $E_{n}(V)$ have been given by Dwyer [8], Bárány and Larman [1], [2], and Buchta [7]. Dwyer also pointed out the role of $E_{n}(v)$ and $E_{n}(f)$ in the design and analysis of algorithms concerning the construction of convex hulls. Recently, van Wel [18] obtained the asymptotic expansions of $E_{n}(f)$ and $E_{n}(v)$ for a $d$-cube, where the asymptotic constants are expressed by means of quite complicated integrals whose explicit determination seems to become hard for $d \geqq 4$. Van Wel [18] also sketched an extension to simple $d$-polytopes, but it is not obvious how one should proceed exactly.

Further results and references about the convex hull of random points and related problems can be found in the surveys of Buchta [5] and Schneider [17].

In the present paper we extend the results mentioned above in the following sense. For a simple $d$-polytope in $\mathbb{R}^{d}(d \geqq 1)$ with $r$ vertices we prove

$$
\begin{aligned}
& E_{n}(f)=\frac{r d^{d}}{d !} M_{1}\left(\Delta_{d-1}\right) \log ^{d-1} n+O\left(\log ^{d-2} n\right), \\
& E_{n}(v)=\frac{r d}{(d+1)^{d-1}} \log ^{d-1} n+O\left(\log ^{d-2} n\right),
\end{aligned}
$$

and

$$
E_{n}(V)=V(P)-\frac{r d V(P)}{(d+1)^{d-1}} \frac{\log ^{d-1} n}{n}+O\left(\frac{\left(\log ^{d-2} n\right.}{n}\right)
$$

where $M_{1}\left(\Delta_{d-1}\right)$ is the first normalized moment of the volume of a random simplex in a $(d-1)$-dimensional simplex. Further, we also give some results concerning the shape of $P_{n}$ (Propositions 1 and 2) and in particular the position of the vertices of $P_{n}$ (Proposition 3). The main tool in our proofs is a class of affine invariant functionals introduced in [20]. We show that they can be related asymptotically to 
$E_{n}(f), E_{n}(v)$, and $E_{n}(V)$. This approach enables us to avoid a separate study of the functionals in question.

For definitions and classical tools in integral geometry and the theory of convex polytopes see the books by Santaló [16] and Brondsted [3], respectively.

\section{The Results}

First we have to define the class of functionals mentioned in the introduction. Let $Q$ be a (convex) polytope contained in $P$ and let $F(Q)$ be the set of facets of $Q$. For $F \in F(Q)$ let $\lambda_{d-1}(F)$ be the $(d-1)$-dimensional volume of $F$, and denote by $\gamma_{F}$ the distance between the affine hull aff $F$ of $F$ and the supporting hyperplane of $P$ parallel to $F$ and separated from $Q$ by aff $F$. Finally, for $q \geqq 0$ define

$$
T_{q}(Q):=\sum_{F \in F(Q)} \gamma_{F}^{q} \lambda_{d-1}^{q}(F)
$$

In the case of a simple $d$-polytope $P$ in $\mathbb{R}^{d}$, an asymptotic expression for $E_{n}\left(T_{q}\right)$ involving the $k$ th normalized moment of the volume of a random simplex in a $(d-1)$-dimensional simplex can be obtained. More precisely, for a $(d-1)$-dimensional convex body $K$ in $\mathbb{R}^{d-1}$ and for $k \in \mathbb{N}$ define

$$
M_{k}(K)=\lambda_{d-l}^{-d-k}(K) \int_{K^{d}} \lambda_{d-1}^{k}\left(\operatorname{conv}\left\{X_{1}, \ldots, X_{d}\right\}\right) d \lambda_{d-1}^{d \otimes}\left(X_{1}, \ldots, X_{d}\right),
$$

where $\lambda_{d-1}$ denotes the Lebesque measure in $\mathbb{R}^{d-1}$. Further, let $\Delta_{d-1}$ be any $(d-1)$-dimensional simplex in $\mathbb{R}^{d-1}$. Since $M_{k}$ is invariant with respect to affine transformations, $M_{k}\left(\Delta_{d-1}\right)$ does not depend on the particular choice of the simplex $\Delta_{d-1}$. We shall prove the following result.

Theorem. If $P$ is a simple d-polytope in $\mathbb{R}^{d}$ with $r$ vertices, then

$$
E_{n}\left(T_{q}\right)=V(P)^{q} \frac{r(d+q-1) ! d^{d+q-1}}{((d-1) !)^{2}} M_{q+1}\left(\Delta_{d-1}\right) \frac{\log ^{d-1} n}{n^{q}}+O\left(\frac{\log ^{d-2} n}{n^{q}}\right)
$$

for each integer $q \geqq 0$ as $n$ tends to infinity.

It can be readily verified that $T_{0}=f$. Hence the following corollary follows immediately from the Theorem.

Corollary 1. For a simple d-polytope $P$ in $\mathbb{R}^{d}$ with $r$ vertices, we have

$$
E_{n}(f)=\frac{r d^{d}}{d !} M_{1}\left(\Delta_{d-1}\right) \log ^{d-1} n+O\left(\log ^{d-2} n\right)
$$

as $n$ tends to infinity. 
To describe the relation between $E_{n}\left(T_{1}\right), E_{n}(V)$ and $E_{n}(v)$ we need another functional which seems to be of interest in its own right. For any polytope $Q$ in $\mathbb{R}^{d}$ and any $x \in Q$ let

$$
N(Q, x):=\left\{y \in \mathbb{R}^{d} \mid\langle y, z-x\rangle \leqq 0 \text { for all } z \in Q\right\}
$$

be the convex cone of all normal vectors of $Q$ at $x$, and let $\tilde{v}(Q)$ be the number of vertices $x$ of $Q$ such that $N(Q, x) \subset N(P, y)$ for some vertex $y$ of $P$. Between $E_{n}\left(T_{1}\right), E_{n}(V)$, and $E_{n+1}(v-\tilde{v})$ we have the following inequalities.

Proposition 1. If $P$ is a simple $d$-polytope in $\mathbb{R}^{d}$ and $n \geqq d+1$, then

$$
\frac{1}{d} E_{n}\left(T_{1}\right) \leqq V(P)-E_{n}(V) \leqq \frac{1}{d} E_{n}\left(T_{1}\right)+V(P) \frac{E_{n+1}(v-\tilde{v})}{n+1}
$$

The next proposition shows that $V(P)-E_{n}(V) \sim(1 / d) E_{n}\left(T_{1}\right)$ as $n$ tends to infinity.

Proposition 2. For a simple d-polytope $P$ in $\mathbb{R}^{d}$, we have

$$
E_{n}(v-\tilde{v})=O\left(\log ^{d-2} n\right)
$$

as $n$ tends to infinity.

While the explicit value of $M_{1}\left(\Delta_{d-1}\right)$ is still unknown for $d \geqq 4$ (see the discussion in [12] and [11] for a lower bound), Reed [13] obtained

$$
M_{2}\left(\Delta_{d-1}\right)=\frac{(d-1) !}{d^{d-1}(d+1)^{d-1}}
$$

Hence, combining Proposition 1, Proposition 2, and the Theorem, we get the following expression for $E_{n}(V)$.

Corollary 2. If $P$ is a simple d-polytope in $\mathbb{R}^{d}$ with $r$ vertices, then

$$
E_{n}(V)=V(P)-V(P) \frac{r d}{(d+1)^{d-1}} \frac{\log ^{d-1} n}{n}+O\left(\frac{\log ^{d-2} n}{n}\right)
$$

as $n$ tends to infinity.

Now by Efron's relation [9],

$$
\frac{E_{n}(v)}{n}=1-\frac{E_{n-1}(V)}{V(P)}, \quad n \geqq d+2,
$$

the next corollary is an obvious consequence of the Theorem, Proposition 2, and Corollary 2. 
Corollary 3. For a simple d-polytope $P$ in $\mathbb{R}^{d}$ with $r$ vertices, we have

$$
E_{n}(v)=\frac{r d}{(d+1)^{d-1}} \log ^{d-1} n+O\left(\log ^{d-2} n\right)
$$

and

$$
E_{n}(\tilde{v})=\frac{r d}{(d+1)^{d-1}} \log ^{d-1} n+O\left(\log ^{d-2} n\right)
$$

as $n$ tends to infinity.

Corollary 3 implies that $E_{n}(v) \sim E_{n}(\tilde{v})$ as $n$ tends to infinity. Another result of this type concerning the position of the vertices of $P_{n}$ seems to be of interest. For any polytope $Q$ contained in $P$ and any $\varepsilon>0$ let $v_{\varepsilon}(Q)$ be the number of vertices $x$ of $Q$ such that $|x-y|<\varepsilon$ for some vertex $y$ of $P$, where $|\cdot|$ denotes the Euclidean norm.

Proposition 3. For a simple d-polytope $P$ in $\mathbb{R}^{d}$ with $r$ vertices and for $\varepsilon>0$ we have

$$
E_{n}\left(v_{\varepsilon}\right)=\frac{r d}{(d+1)^{d-1}} \log ^{d-1} n+O\left(\log ^{d-2} n\right)
$$

and hence $E_{n}\left(v_{\varepsilon}\right) \sim E_{n}(v)$ as $n$ tends to infinity.

Remark 1. For simplicial polytopes with a large number of vertices the extreme values of $f / v$ given in the lower and upper bound theorems (see [3]) are far from each other. Hence it seems worth noticing that for a simple $d$-polytope $P$ in $\mathbb{R}^{d}$, Corollaries 1 and 3 together imply that

$$
\lim _{n \rightarrow \infty} \frac{E_{n}(f)}{E_{n}(v)}=\left(\frac{1}{d}\right) \frac{M_{1}\left(\Delta_{d-1}\right)}{M_{2}\left(\Delta_{d-1}\right)},
$$

since this equality provides some information about the shape of $P_{n}$ (for large values of $n$ ) in the "average case". In [20] a similar expression was obtained for the corresponding limit in the case where $P$ is replaced by a ball in $\mathbb{R}^{d}$, namely

$$
\frac{2 d(d+3)}{\left(d^{2}+1\right)\left(d^{2}+d+2\right)} \frac{M_{1}\left(B_{d-1}\right)}{M_{2}\left(B_{d-1}\right)} .
$$

Here $B_{d-1}$ is the unit ball of $\mathbb{R}^{d-1}$.

Remark 2. For any $d$-polytope $Q$ contained in $P$ let

$$
S_{P}(Q):=\lim _{\varepsilon \rightarrow 0} \frac{V(Q+\varepsilon P)-V(Q)}{\varepsilon}
$$


be the Minkowski area of $Q$ relative to $P$. Then it is not difficult to see that

$$
T_{1}(Q)=S_{P}(Q)-d V(Q)
$$

Hence, if $P$ is a simple $d$-polytope in $\mathbb{R}^{d}$, Propositions 1 and 2 imply that

$$
V(P)-\frac{1}{d} E_{n}\left(S_{P}\right)=O\left(\frac{\log ^{d-2} n}{n}\right)
$$

as $n$ tends to infinity.

\section{Proof of the Results}

We shall need the following lemma.

Lemma. For all integers $r, s \geqq 0$ and $d \geqq 2$ and for all $c \in(0,1]$

$$
\begin{aligned}
I: & :=\int_{0}^{1} \cdots \int_{0}^{1}\left(1-c x_{1} \cdots x_{d}\right)^{n-s}\left(x_{1} \cdots x_{d}\right)^{r} d x_{1} \cdots d x_{d} \\
& =\frac{r !}{(d-1) ! c^{r+1}} \frac{\log ^{d-1} n}{n^{r+1}}+O\left(\frac{\log ^{d-2} n}{n^{r+1}}\right)
\end{aligned}
$$

as $n$ tends to infinity.

Proof. Following Dwyer [8], we consider the distribution function $F$ defined by

$$
F(y)=\lambda_{d}\left(\left\{\left(x_{1}, \ldots, x_{d}\right) \in[0,1]^{d} \mid x_{1} \ldots x_{d} \leqq y\right\}\right), \quad 0<y<1 .
$$

The density function of $F$ is

$$
\frac{1}{(d-1) !} \log ^{d-1}\left(\frac{1}{y}\right)
$$

(see $\S I .8$ of [10] and the comment by Dwyer on p. 691 of [8]). Hence, for large $n$, we have

$$
\begin{aligned}
I & =\frac{1}{(d-1) !} \int_{0}^{1}(1-c y)^{n-s} y^{r} \log ^{d-1}\left(\frac{1}{y}\right) d y \\
& =\frac{1}{(d-1) !} \frac{1}{(c(n-s))^{r+1}} \int_{0}^{c(n-s)}\left(1-\frac{z}{n-s}\right)^{n-s} z^{r}(\log c(n-s)-\log z)^{d-1} d z \\
& =\sum_{k=0}^{d-1} \frac{1}{k !(d-1-k) !} \frac{\log ^{d-1-k} c(n-s)}{c^{r+1}(n-s)^{r+1}} I_{k}(n),
\end{aligned}
$$


where

$$
I_{k}(n)=\int_{0}^{c(n-s)}\left(1-\frac{z}{n-s}\right)^{n-s} z^{r}(-\log z)^{k} d z
$$

Here we have used the substitution $z=c(n-s) y$. Now, a slight modification of the argument given on p. 242 of [19], shows that $I_{0}(n)=r !+O(1 / n)$. For $k=1, \ldots, d-1$ the absolute value of the integrand in $I_{k}(n)$ is majorized by $e^{-z} z^{r+k}$ for $1 \leqq z \leqq c(n-s)$ and by $z^{r}|\log z|^{k}$ for $0<z<1$. Hence, for $k=1, \ldots, d-1$ there is a $c_{k}>0$ such that $I_{k}(n)<c_{k}$ for all $n \in \mathbb{N}$. Finally, it is easy to see that

$$
\frac{\log ^{p} c(n-s)}{(n-s)^{r+1}}=\frac{\log ^{p} n}{n^{r+1}}+o\left(\frac{\log ^{p-1} n}{n^{r+1}}\right)
$$

as $n$ tends to infinity, which completes the proof.

With a similar argument it is easy to see that for $d=1$

$$
I=\frac{r !}{(c n)^{r+1}}+O\left(\frac{1}{n^{r+2}}\right)
$$

as $n$ tends to infinity. We are now in a position to prove the Theorem.

Proof of the Theorem. For $\lambda_{d}^{d \otimes}$-almost all $\left(X_{1}, \ldots, X_{d}\right) \in P^{d}$ there is exactly one hyperplane $L\left(X_{1}, \ldots, X_{d}\right)$ through $X_{1}, \ldots, X_{d}$. If $H$ is a hyperplane and $y$ is a vertex of $P$ define

$$
\varepsilon_{y}(H):= \begin{cases}1 & \text { if } H \text { is parallel to a supporting hyperplane of } P \text { through } y \\ 0 & \text { otherwise. }\end{cases}
$$

Further, let $\eta_{y}(H)$ be the distance of $y$ from $H$ and let $\hat{V}(H)$ be the volume of the part of $P$ cut off by $H$ and containing $y$. Now, define

$$
I_{n}(P, y):=\frac{\left(\begin{array}{l}
n \\
d
\end{array}\right)}{V(P)^{q+d}} \int_{P^{d}}\left(1-\frac{\hat{V} \circ L}{V(P)}\right)^{n-d} \sigma_{d-1}^{q}\left(\eta_{y} \circ L\right)^{q}\left(\varepsilon_{y} \circ L\right) d \lambda_{d}^{d \otimes}
$$

where $\sigma_{d-1}\left(X_{1}, \ldots, X_{d}\right)=\lambda_{d-1}\left(\operatorname{conv}\left\{X_{1}, \ldots, X_{d}\right\}\right)$. Then standard arguments show that

$$
E_{n}\left(T_{q}\right)=V(P)^{q} \sum_{y \text { vertex of } P} I_{n}(P, y)
$$

Moreover, for each affine transformation $\varphi$ of $\mathbb{R}^{d}$ we have $I_{n}(\varphi P, \varphi y)=I_{n}(P, y)$. Hence, in order to compute $I_{n}(P, y)$ we may assume that $y$ is the origin 0 and that 
the $d$ vertices adjacent to $y$ are $e_{1}:=(1,0, \ldots, 0), \ldots, e_{d}:=(0, \ldots, 0,1)$. By the Blaschke-Petkantschin identity (see p. 201 of [16]) we have

$$
I_{n}(P, y)=\frac{\left(\begin{array}{l}
n \\
d
\end{array}\right)(d-1) !}{V(P)^{q+d}} \int_{\mathscr{E}_{P}}\left(1-\frac{\hat{V}}{V(P)}\right)^{n-d} \eta_{y}^{q} \varepsilon_{y} J d v
$$

where $\mathscr{E}_{P}$ is the set of all hyperplanes meeting $P, v$ is the usual motion invariant measure on the space of hyperplanes, and for $H \in \mathscr{E}_{P}$

$$
J(H)=\int_{(H \cap P)^{d}} \sigma_{d-1}^{q+1} d \lambda_{d-1}^{d \otimes} .
$$

For any integrable function $f$ on the space of hyperplanes we have

$$
\int f d v=\int_{S^{d-1}} \int_{0}^{\infty} f\left(H_{\tau, u}\right) d \tau d \omega(u)
$$

where $\omega$ is the usual surface area measure on $S^{d-1}$ and

$$
H_{\tau, u}=\left\{x \in \mathbb{R}^{d} \mid\langle x, u\rangle=\tau\right\}
$$

Substituting $z=u / \tau$ and defining $H_{z}:=\left\{x \in \mathbb{R}^{d} \mid\langle x, z\rangle=1\right\}$ for $\tau>0, z \neq 0$, we get

$$
\int f d v=\int_{\mathbb{R}^{d}} f\left(H_{\mathrm{z}}\right)|z|^{-d-1} d \lambda_{d}(z)
$$

Now, for $p=0, \ldots, d$ define

$$
\mathscr{E}^{p}(y):=\left\{H_{z} \in \mathscr{E}_{P} \mid z_{i}>1 \text { for exactly } p \text { coordinates } z_{i} \text { of } z\right\}
$$

(i.e., $\mathscr{E}^{p}(y)$ is the set of all hyperplanes separating $y$ from $p$ of the vertices adjacent to $y$ ) and

$$
I_{n}^{p}(P, y):=\frac{\left(\begin{array}{l}
n \\
d
\end{array}\right)(d-1) !}{V(P)^{q+d}} \int_{\delta^{p}(y)}\left(1-\frac{\hat{V}}{V(P)}\right)^{n-d} \eta_{y}^{q} \varepsilon_{y} J d v
$$

For $H_{z} \in \mathscr{E}^{d}(y)$ and $z=\left(z_{1}, \ldots, z_{d}\right)$ it is easy to see that

$$
\hat{V}\left(H_{z}\right)=\left(d ! z_{1} \cdots z_{d}\right)^{-1}, \quad \eta_{y}\left(H_{z}\right)=|z|^{-1},
$$

and

$$
A_{d-1}\left(H_{z}\right):=\lambda_{d-1}\left(H_{z} \cap P\right)=\frac{d \hat{V}\left(H_{z}\right)}{\eta_{y}\left(H_{z}\right)}=\left((d-1) ! z_{1} \cdots z_{d}\right)^{-1}|z|
$$


Moreover, since $M_{q+1}$ is invariant with respect to affine transformations, we have

$$
J\left(H_{z}\right)=M_{q+1}\left(\Delta_{d-1}\right) A_{d-1}^{d+q+1}\left(H_{z}\right) .
$$

It follows

$$
\begin{aligned}
I_{n}^{d}(P, y)= & \frac{\left(\begin{array}{l}
n \\
d
\end{array}\right)(d-1) !}{V(P)^{q+d}} M_{q+1}\left(\Delta_{d-1}\right) \\
& \times \int_{1}^{\infty} \cdots \int_{1}^{\infty}\left(1-\frac{\hat{V}\left(H_{z}\right)}{V(P)}\right)^{n-d} \eta_{y}^{q}\left(H_{z}\right) A_{d-1}^{d+q+1}\left(H_{z}\right)|z|^{-d-1} d z_{1} \cdots d z_{d} \\
= & \frac{\left(\begin{array}{l}
n \\
d
\end{array}\right)^{M_{q+1}\left(\Delta_{d-1}\right)}}{V(P)^{q+\mathrm{d}}\left((d-\overline{1) !})^{d+q}\right.} \int_{1}^{\infty} \cdots \int_{1}^{\infty}\left(1-\frac{1}{d ! V(P) z_{1} \cdots z_{d}}\right)^{n-d} \\
& \times\left(z_{1} \cdots z_{d}\right)^{-d-q-1} d z_{1} \cdots d z_{d} .
\end{aligned}
$$

Substituting $x_{i}=z_{i}^{-1}$, we get

$$
\begin{aligned}
I_{n}^{d}(P, y)= & \frac{\left(\begin{array}{l}
n \\
d
\end{array}\right) M_{q+1}\left(\Delta_{d-1}\right)}{V(P)^{q+d}((d-1) !)^{d+q}} \\
& \times \int_{0}^{1} \cdots \int_{0}^{1}\left(1-\frac{x_{1} \cdots x_{d}}{d ! V(P)}\right)^{n-d}\left(x_{1} \cdots x_{d}\right)^{d+q-1} d x_{1} \cdots d x_{d}
\end{aligned}
$$

and since $d ! V \geqq 1$, the preceding lemma yields

$$
I_{n}^{d}(P, y)=\frac{(d+q-1) ! d^{d+q-1}}{((d-1) !)^{2}} M_{q+1}\left(\Delta_{d-1}\right) \frac{\log ^{d-1} n}{n^{q}}+O\left(\frac{\log ^{d-2} n}{n^{q}}\right)
$$

We now turn to the estimation of $I_{n}^{p}(P, y)$ for $p=1, \ldots, d-1$. Suppose that $H_{z} \in \mathscr{E}^{p}(y)$ and assume without loss of generality that $z_{1}, \ldots, z_{p}>1$ and $z_{p+1}, \ldots, z_{d} \leqq 1$. Then, $H_{z} \cap[0, \infty)^{d}$ is a simplex $a(p-1)$-side of which is

$$
B\left(z_{1}, \ldots, z_{p}\right):=\left\{\left(x_{1}, \ldots, x_{d}\right) \in[0,1]^{d} \mid x_{1} z_{1}+\cdots+x_{p} z_{p}=1, x_{p+1}=\cdots=x_{d}=0\right\} .
$$

Hence, by the compactness of $P$, there is a constant $\alpha$ depending only on $P, d$, and $p$ such that

$$
\begin{aligned}
\lambda_{d-1}\left(H_{z} \cap P\right) & \leq \alpha \lambda_{p-1}\left(B\left(z_{1}, \ldots, z_{p}\right)\right) \\
& =\alpha\left((p-1) ! z_{1} \cdots z_{p}\right)^{-1}\left(z_{1}^{2}+\cdots+z_{p}^{2}\right)^{1 / 2} \\
& \leqq \alpha\left((p-1) ! z_{1} \cdots z_{p}\right)^{-1}|z|
\end{aligned}
$$


Further, since the points $z_{1}^{-1} e_{1}, \ldots, z_{\mathrm{p}}^{-1} e_{p}, e_{p+1}, \ldots, e_{d}$ lie in the halfspace bounded by $H_{z}$ and containing $y, \hat{V}\left(H_{z}\right)$ is not smaller than the volume of the convex hull of these points, which implies

$$
\widehat{V}\left(H_{z}\right) \geqq\left(d ! z_{1} \cdots z_{p}\right)^{-1}
$$

These arguments show that there is a constant $\beta$ depending only on $P, d$, and $p$ such that

$$
\begin{aligned}
I_{n}^{p}(P, y) \leqq\left(\begin{array}{l}
n \\
d
\end{array}\right) \beta \int_{0}^{1} \cdots \int_{0}^{1}( & \int_{1}^{\infty} \cdots \int_{1}^{\infty}\left(1-\frac{1}{d ! V(P) z_{1} \cdots z_{p}}\right)^{n-d} \\
& \left.\quad \times\left((p-1) ! z_{1} \cdots z_{p}\right)^{-d-q-1} d z_{1} \cdots d z_{p}\right) d z_{p+1} \cdots d z_{d}
\end{aligned}
$$

Using the substitution $x_{i}=z_{i}^{-1}$ (for $i=1, \ldots, p$ ) as above and the Lemma, we conclude that

$$
I_{n}^{p}(P, y)=O\left(\frac{\log ^{p-1} n}{n^{q}}\right)
$$

Finally, for $H_{z} \in \mathscr{E}^{\circ}(y)$ we have $\hat{V}\left(H_{z}\right)>1 / d !$ and hence

$$
I_{n}^{0}(P, y)=O\left(n^{d}\left(1-\frac{1}{d ! V(P)}\right)^{n-d}\right)
$$

Now, the assertion follows from (1), (2), (3), and (4) together.

Proof of Proposition 1. For $X_{1}, \ldots, X_{n} \in P$ let $Q_{n}\left(X_{1}, \ldots, X_{n}\right)$ be the union of all sets of the form $\operatorname{conv}(\{y\} \cup F)$ where $y$ is a vertex of $P$ and $F$ is a facet of $P_{n}\left(X_{1}, \ldots, X_{n}\right)$ such that the outer unit normal vector of $P_{n}\left(X_{1}, \ldots, X_{n}\right)$ at $F$ belongs to $N(P, y)$. Then, it is easy to see that

$$
\lambda_{d}\left(Q_{n}\right)=\frac{1}{d} T_{1} \quad \text { and } \quad Q_{n} \subset P \backslash P_{n},
$$

which implies $(1 / d) E_{n}\left(T_{1}\right) \leqq V(P)-E_{n}(V)$. We now turn to the proof of the second inequality. If $X \in P \backslash P_{n}\left(X_{1}, \ldots, X_{n}\right)$ satisfies

$$
N\left(P_{n+1}\left(X, X_{1}, \ldots, X_{n}\right), X\right) \subset N(P, y)
$$

for some vertex $y$ of $P$, then all facets of $P_{n+1}\left(X, X_{1}, \ldots, X_{n}\right)$ containing $X$ are parallel to a supporting hyperplane of $P$ at $y$, and it can be readily verified that 
$X \in Q\left(X_{1}, \ldots, X_{n}\right)$. Hence, defining

$$
\varepsilon\left(X, X_{1}, \ldots, X_{n}\right)= \begin{cases}1 & \text { if } X \in P \backslash\left(P_{n}\left(X_{1}, \ldots, X_{n}\right) \cup Q_{n}\left(X_{1}, \ldots, X_{n}\right)\right) \\ 0 & \text { otherwise }\end{cases}
$$

and for $i=1, \ldots, n+1$

$$
\delta_{i}\left(X_{1}, \ldots, X_{n+1}\right)= \begin{cases}1 & \text { if } N\left(P_{n+1}\left(X_{1}, \ldots, X_{n+1}\right), X_{i}\right) \not \subset N(P, y) \\ & \text { for each vertex } y \text { of } P \\ 0 & \text { otherwise }\end{cases}
$$

we have $\varepsilon\left(X, X_{1}, \ldots, X_{n}\right) \leqq \delta_{1}\left(X, X_{1}, \ldots, X_{n}\right)$ for all $X, X_{1}, \ldots, X_{n} \in P$. This implies

$$
\begin{aligned}
E_{n}\left(\lambda_{d}(P \backslash\right. & \left.\left.\left(P_{n} \cup Q_{n}\right)\right)\right) \\
& =V(P)^{-n} \int_{P^{n}}\left(\int_{P} \varepsilon\left(X, X_{1}, \ldots, X_{n}\right) d \lambda_{d}(X)\right) d \lambda_{d}^{n \otimes}\left(X_{1}, \ldots, X_{n}\right) \\
& \leqq V(P)^{-n} \int_{P^{n+1}} \delta_{1}\left(X_{1}, \ldots, X_{n+1}\right) d \lambda_{d}^{(n+1) \otimes}\left(X_{1}, \ldots, X_{n+1}\right) \\
& =\frac{1}{n+1} V(P)^{-n} \int_{P^{n+1}} \sum_{i=1}^{n+1} \delta_{i}\left(X_{1}, \ldots, X_{n+1}\right) d \lambda_{d}^{(n+1) \otimes}\left(X_{1}, \ldots, X_{n+1}\right) \\
& =\frac{V(P)}{n+1} E_{n+1}(v-\tilde{v}) .
\end{aligned}
$$

Now the second inequality follows from (5), which completes the proof of Proposition 1.

Proof of Proposition 2. First remark that for any polytope $Q$, any $k$-face $F$ of $Q$ $(1 \leqq k \leqq d-1)$, and any $x, y$ belonging to the relative interior of $F$ we have $N(Q, x)=N(Q, y)$, i.e., $N(Q, x)$ does not depend on the particular choice of $x$, and we denote this convex cone by $N(Q, F)$. Now, suppose that $Q \subset P$ and $x$ is a vertex of $Q$ with $N(Q, x) \not \subset N(P, y)$ for each vertex $y$ of $P$. Then there are at least two vertices $y$ and $\bar{y}$ of $P$ such that $N(Q, x)$ meets the interior of $N(P, y)$ and $N(P, \bar{y})$. Since $N(Q, x)$ is convex and has interior points, there is an edge $K$ of $P$ such that $N(Q, x)$ meets the relative interior of $N(P, K)$. Hence, denoting by $\tilde{v}_{K}(Q)$ the number of all vertices $x$ of $Q$ such that $N(Q, x)$ meets the relative interior of $N(P, K)$, we have

$$
v(Q)-\tilde{v}(Q) \leqq \sum_{\boldsymbol{K} \text { edge of } \boldsymbol{P}} \tilde{v}_{K}(Q)
$$


Thus, to prove $E_{n}(v-\tilde{v})=O\left(\log ^{d-2} n\right)$, it suffices to show that $E_{n}\left(\tilde{v}_{K}\right)=O\left(\log ^{d-2} n\right)$ for each edge $K$ of $P$. Let $K$ be an edge of $P$. It is easy to see that $E_{n}\left(\tilde{v}_{K}\right)$ is invariant with respect to affine transformations of $\mathbb{R}^{d}$. Hence, we may assume that 0 is a vertex of $P$, that the vectors $e_{1}, \ldots, e_{d}$ of the standard basis of $\mathbb{P}^{d}$ are the vertices adjacent to 0 , and that $K$ is the edge joining 0 and $e_{d}$. For $i=1, \ldots, n$ and $X_{1}, \ldots, X_{n} \in P$ define

$$
\varepsilon_{i}\left(X_{1}, \ldots, X_{n}\right):=\left\{\begin{array}{cc}
1 & \begin{array}{l}
\text { if } X_{i} \text { is a vertex of } P_{n}\left(X_{1}, \ldots, X_{n}\right) \text { and } \\
N\left(P_{n}\left(X_{1}, \ldots, X_{n}\right), X_{i}\right) \text { meets the relative } \\
\text { interior of } N(P, K) \\
\text { otherwise. }
\end{array}
\end{array}\right.
$$

Suppose that $\varepsilon_{1}\left(X_{1}, \ldots, X_{n}\right)=1$ and that $u \in N\left(P_{n}\left(X_{1}, \ldots, X_{n}\right), X_{1}\right)$ belongs to the relative interior of $N(P, K)$. Then we have

$$
\begin{gathered}
\left\langle u, e_{j}\right\rangle \leqq 0 \quad \text { for all } j \in\{1, \ldots, d-1\}, \quad\left\langle u, e_{d}\right\rangle=0, \\
x_{j}:=\left\langle X_{1}, e_{j}\right\rangle \geqq 0 \quad \text { for all } j \in\{1, \ldots, d\}
\end{gathered}
$$

and hence

$$
\begin{aligned}
\left\langle u, x_{i} e_{i}-X_{1}\right\rangle & =x_{i}\left\langle u, e_{i}\right\rangle-\sum_{j=1}^{d} x_{j}\left\langle u, e_{j}\right\rangle \\
& =-\sum_{\substack{j=1 \\
j \neq i}}^{d} x_{j}\left\langle u, e_{j}\right\rangle \geqq 0
\end{aligned}
$$

for $i=1, \ldots, d-1$. This implies that $\operatorname{conv}\left\{0, x_{1} e_{1}, \ldots, x_{d-1} e_{d-1}, e_{d}\right\}$ and $P_{n}\left(X_{1}, \ldots, X_{n}\right)$ are separated by the hyperplane orthogonal to $u$ through $X_{1}$. Thus, denoting by $C\left(X_{1}\right)$ the interior of

$$
\operatorname{conv}\left\{0, \min \left\{x_{1}, 1\right\} e_{1}, \ldots, \min \left\{x_{d-1}, 1\right\} e_{d-1}, e_{d}\right\},
$$

we have $C\left(X_{1}\right) \subset P$ and $X_{2}, \ldots, X_{n} \notin C\left(X_{1}\right)$. It follows that

$$
\begin{aligned}
E_{n}\left(\tilde{v}_{K}\right) & =V(P)^{-n} \int_{P^{n}} \sum_{i=1}^{n} \varepsilon_{i} d \lambda_{d}^{n \otimes}=n V(P)^{-n} \int_{P^{n}} \varepsilon_{1} d \lambda_{d}^{n \otimes} \\
& \leqq \frac{n}{V(P)} \int_{P}\left(1-\frac{V\left(C\left(X_{1}\right)\right)}{V(P)}\right)^{n-1} d \lambda_{d}\left(X_{1}\right) \\
& =\frac{n}{V(P)} \sum_{p=0}^{d-1} \int_{R^{p}}\left(\frac{1-V\left(C\left(X_{1}\right)\right)}{V(P)}\right)^{n-1} d \lambda_{d}\left(X_{1}\right),
\end{aligned}
$$

where

$R^{p}:=\left\{x \in P \mid x_{i} \leqq 1\right.$ for exactly $p$ of the first $d-1$ coordinates $x_{i}$ of $\left.x\right\}$. 
Now, for $p \in\{1, \ldots, d-1\}$ and $X_{1}:=\left(x_{1}, \ldots, x_{d}\right) \in R^{p}$ with $x_{1}, \ldots, x_{p} \leqq 1$ and $x_{p+1}, \ldots, x_{d-1}>1$, e.g., we have $V\left(C\left(X_{1}\right)\right)=(1 / d !) x_{1} \cdots x_{p}$, hence there is a constant $c_{p}>0$ such that

$$
\begin{aligned}
\int_{R^{p}}\left(1-\frac{V\left(C\left(X_{1}\right)\right)}{V(P)}\right)^{n-1} d \lambda_{d}\left(X_{1}\right) & \leqq c_{p} \int_{0}^{1} \cdots \int_{0}^{1}\left(1-\frac{x_{1} \cdots x_{p}}{d !}\right)^{n-1} d x_{1} \cdots d x_{p} \\
& =O\left(\frac{\log ^{p-1} n}{n}\right)
\end{aligned}
$$

where the last relation follows from the Lemma. Further, for $X_{1} \in R^{0}$ we have $V\left(C\left(X_{1}\right)\right)=1 / d$ ! and hence $n$ times the integral over $R^{0}$ in (6) converges to 0 as $n$ tends to infinity. It follows that

$$
E_{n}\left(\tilde{v}_{K}\right)=O\left(\log ^{d-2} n\right)
$$

which completes the proof.

Proof of Proposition 3. For any polytope $Q$ contained in $P$ and any vertex $y$ of $P$ define

$$
W_{y, \varepsilon}(Q):=\left\{x \in \mathbb{R}^{d} \mid x \text { is a vertex of } Q, N(Q, x) \subset N(P, y),|x-y| \geqq \varepsilon\right\},
$$

and for $A \subset \mathbb{R}^{d}$ let $|A|$ denote the cardinality of $A$. We shall prove that $E_{n}\left(\left|W_{y, \varepsilon}\right|\right)=O\left(\log ^{d-2} n\right)$ for each vertex $y$ of $P$, the assertion then follows from Corollary 3 . Hence, let $y$ be a vertex of $P$, without loss of generality $y=0$. Further, let $b_{1}, \ldots, b_{d}$ be the vertices of $P$ adjacent to $y=0$. For $0<\alpha<1$ and $j=1, \ldots, d$ define

$$
\begin{aligned}
W_{\alpha}^{j}\left(P, b_{1}, \ldots, b_{d}, Q\right) \\
\quad:=\left\{x=\sum_{i=1}^{d} x_{i} b_{i} \mid x \text { is a vertex of } Q, N(Q, x) \subset N(P, y), x_{j}>\alpha\right\}
\end{aligned}
$$

and $U_{\alpha}^{j}(Q):=W_{\alpha}^{j}\left(P, b_{1}, \ldots, b_{d}, Q\right)$. If $\alpha$ is small enough, then

$$
W_{y, \varepsilon}(Q) \subset \bigcup_{j=1}^{d} U_{\alpha}^{j}(Q),
$$

and for any injective linear map $\varphi: \mathbb{R}^{d} \rightarrow \mathbb{R}^{d}$ we have

$$
W_{\alpha}^{j}\left(\varphi(P), \varphi\left(b_{1}\right), \ldots, \varphi\left(b_{d}\right), \varphi(Q)\right)=\varphi\left(W_{\alpha}^{j}\left(P, b_{1}, \ldots, b_{d}, Q\right)\right)
$$

Hence, to prove that $E_{n}\left(\left|W_{y, \varepsilon}\right|\right)=O\left(\log ^{d-2} n\right)$, it suffices to show that $E_{n}\left(\left|U_{\alpha}^{j}\right|\right)=$ $O\left(\log ^{d-2} n\right)(n \rightarrow \infty)$ for $j=1, \ldots, d$. For this purpose we may assume that 
$\left\{b_{1}, \ldots, b_{d}\right\}$ is the standard basis of $\mathbb{R}^{d}$. Now, as in the proof of Proposition 2, $X_{1}, \ldots, X_{n} \in P$ and $X_{1} \in U_{\alpha}^{j}\left(P_{n}\left(X_{1}, \ldots, X_{n}\right)\right)$ imply that

$$
\operatorname{conv}\left\{0, x_{1} b_{1}, \ldots, x_{j-1} b_{j-1}, \alpha b_{j}, x_{j+1} b_{j+1}, \ldots, x_{d} b_{d}\right\}
$$

and $P_{n}\left(X_{1}, \ldots, X_{n}\right)$ are separated by a supporting hyperplane of $P_{n}\left(X_{1}, \ldots, X_{n}\right)$ through $X_{1}$. Hence, the argument used in the proof of Proposition 2 shows that

$$
E_{n}\left(\left|U_{\alpha}^{j}\right|\right)=O\left(\log ^{d-2} n\right)
$$

for $j=1, \ldots, d$ as $n$ tends to infinity, which completes the proof.

\section{Acknowledgments}

The authors are indebted to Professor Rolf Schneider (Freiburg i. Br.) for many helpful discussions.

\section{References}

1. I. Bárány, Intrinsic volumes and f-vectors of random polytopes, Math. Ann. 285 (1989), 671-699.

2. I. Bárány and D. G. Larman, Convex bodies, economic cap coverings, random polytopes, Mathematika 35 (1988), 274-291.

j. A. Brøndsted, An introduction to Convex Polytopes, Springer-Verlag, New York, 1982.

4. C. Buchta, Stochastische Approximation konvexer Polygone, Z. Warsch. Verw. Gebiete 67 (1984), $283-304$.

5. C. Buchta, Zufällige Polyeder-Eine Ubersicht, in Zahlentheoretische Analysis (ed. by E. Hlawka), Lecture Notes in Mathematics, Vol. 1114, Springer-Verlag, Berlin, 1985, pp. 1-13.

6. C. Buchta, A note on the volume of a random polytope in a tetrahedron, Illinois J. Math. 30 (1986), 653-659.

7. C. Buchta, A remark on random approximation of simple polytopes, Anz. Österreich. Akad. Wiss. Math.-Natur. Kl. (1989), 17-20.

8. R. A. Dwyer, On the convex hull of random points in a polytope, J. Appl. Probab. 25 (1988), 688-699.

9. B. Efron, The convex hull of a random set of points, Biometrika 52 (1965), 331-343.

10. W. Feller, An Introduction to Probability Theory and its Applications, Vol. 2, 2nd edn., Wiley, New York, 1971.

11. H. Groemer, On some mean values associated with a randomly selected simplex in a convex set, Pacific J. Math. 45 (1973), 525-533.

12. V. Klee, What is the expected volume of a simplex whose vertices are chosen at random from a given convex body? Amer. Math. Monthly 76 (1969), 286-288.

13. W. J. Reed, Random points in a simplex, Pacific J. Math. 54 (1974), 183-198.

14. A. Rényi and R. Sulanke, Über die konvexe Hülle von $n$ zufällig gewählten Punkten, Z. Wahrsch. Verw. Gebiete 2 (1963), 75-84.

15. A. Rényi and R. Sulanke, Über die konvexe Hülle von $n$ zufällig gewählten Punkten, II, Z. Wahrsch. Verw. Gebiete 3 (1964), 138-147.

16. L. A. Santaló, Integral Geometry and Geometric Probability, Addison-Wesley, Reading, Massachusetts, 1976.

17. R. Schneider, Random approximation of convex sets, J. Microscopy 151 (1988), $211-227$. 
18. B. F. van Wel, The convex hull of a uniform sample from the interior of a simple $d$-polytope, J. Appl. Probab. 27 (1989), 259273.

19. E. T. Whittaker and G. N. Watson, A Course of Modern Analysis, 4th edn., Cambridge University Press, Cambridge, 1952.

20. J. A. Wieacker, Einige Probleme der polyedrischen Approximation, Diplomarbeit, Freiburg i. Br., 1978.

Received March 6, 1989, and in revised form May 5, 1989. 